\title{
The $c$-function for non-compactly causal symmetric spaces
}

\author{
Bernhard Krötz* and Gestur Ólafsson**
}

\section{Introduction}

In this paper we prove a product formula for the $c$-function associated to a non-compactly causal symmetric space $\mathcal{M}$. Let us recall here the basic facts. Let $G$ be a connected semisimple Lie group, $\tau: G \rightarrow G$ be a non-trivial involution and $H=G^{\tau}$. Then $\mathcal{M}:=G / H$ is a semsimple symmetric space. The space $\mathcal{M}$ is called non-compactly causal, if $\mathfrak{q}:=\{X \in \mathfrak{g}: \tau(X)=-X\}$ contains an open $H$-invariant hyperbolic cone $C \neq \varnothing$. In this case $S:=H \exp (C)$ is a open subsemigroup of $G$. A spherical function on $\mathcal{M}$ is an $H$-biinvariant continuous function on $S / H \subseteq \mathcal{M}$, which defines an eigendistribution of the algebra of $H$-invariant differential operators on $\mathcal{M}$, see [FHÓ94], [KNÓ98], [Ól97]. There exists a maximal abelian hyperbolic subspace $\mathfrak{a}$ of $\mathfrak{q}$ such that $C=\operatorname{Ad}(H) \cdot(\mathfrak{a} \cap C)$. Let

$$
\varphi_{\lambda}\left(g \cdot x_{0}\right)=\int_{H} a_{H}(g h)^{\lambda-\rho} d \mu_{H}(h)
$$

be a spherical function given by a convergent integral similar to the expression for the spherical functions on the Riemannian symmetric spaces $G / K$. Here $x_{0} \in \mathcal{M}$ is the coset $\{H\}$ and $a_{H}(g) \in A:=\exp (\mathfrak{a})$ is determined by $g \in H a_{H}(g) N$. The asypmtotic behaviour of $\varphi_{\lambda}\left(a . x_{0}\right)$ along $S \cap A$ is given by $\varphi_{\lambda}\left(a . x_{0}\right) \sim c(\lambda) a^{\lambda-\rho}$, where $\rho$ is half the sum over the positive roots counted with multiplicities. The function $c(\lambda)$ is the $c$-function of the space $\mathcal{M}$. It turns out that the $c$-function is a product of two $c$-function, $c(\lambda)=c_{\Omega}(\lambda) c_{0}(\lambda)$ where $c_{0}(\lambda)$ is the HarishChandra $c$-function of a Riemannian subsymmetric space $G(0) / K(0)$ and $c_{\Omega}(\lambda)$ is a function associated to the real bounded symmetric domain $H /(H \cap K)$, where $K$ is a $\tau$-stable maximal compact subgroup of $G$. The $c$-function was first introduced by Oshima-Sekiguchi in [OS80], whereas $c_{\Omega}(\lambda)$ was first introduced in [FHÓ94].

The $c$-function for a Riemannian symmetric space $G / K$ can be written as a product of $c$-functions of rank one symmetric spaces associated to each restricted root of $\mathfrak{g}$ (GindikinKarpelevic formula). For general non-Riemannian symmetric spaces $G / H$ one cannot expect this type of result. However, for non-compactly causal symmetric spaces we show in this paper (cf. Theorem III.5) that such a product formula holds. The case of Cayley type spaces has already been treated by J. Faraut in [Fa95] by the use of Jordan algebra methods and in [Gr97] the case $\mathrm{Sl}(n, \mathbb{R}) / \mathrm{SO}(p, q)$ is dealed. The approach presented here is general, different and relies on new insights on the fine convex geometry of the real bounded symmetric domain $\Omega$ (cf. Theorem II.5 and Theorem II.7.)

Our result has important applications. The $c$-function was the last unknown part in the formula for the formal degree of the spherical holomorphic discrete series representations

\footnotetext{
* Supported by the DFG-project HI 412/5-2

** Supported by LEQSF grant (1996-99)-RD-A-12
} 
representations (cf. [Kr99]). Further it gives us important information on the normalized spherical functions $\widetilde{\varphi}_{\lambda}:=c_{\Omega}(\lambda)^{-1} \varphi_{\lambda}$. One knows that the function $\lambda \mapsto \widetilde{\varphi}_{\lambda}\left(s . x_{0}\right)$ has a meromorphic continuation to $\mathfrak{a}_{\mathbb{C}}^{*}$ (cf. [O197]) and the product formula gives us important information on the poles. In particular, this allows more detailed analysis of the spherical Laplace transform, in particular Paley-Wiener type theorems.

\section{Non-compactly causal symmetric spaces and Lie algebras}

In this section we introduce notation and recall some facts concerning non-compactly causal symmetric Lie algebras and their associated symmetric spaces. Our source of reference is [HiÓl96].

\section{Algebraic preliminaries}

Let $\mathfrak{g}$ be a simple finite dimensional real Lie algebra. Let $\tau: \mathfrak{g} \rightarrow \mathfrak{g}$ be a non-trivial involution. Then $(\mathfrak{g}, \tau)$ is a symmetric Lie algebra. We write $\mathfrak{g}=\mathfrak{h}+\mathfrak{q}$ for the $\tau$-eigenspace decomposition of $\mathfrak{g}$ corresponding to the eigenvalues +1 and -1 . Let $\theta$ be a Cartan involution of $\mathfrak{g}$ which commutes with $\tau$ and let $\mathfrak{g}=\mathfrak{k}+\mathfrak{p}$ be the associated Cartan decomposition.

For $\mathfrak{a}, \mathfrak{b} \subseteq \mathfrak{g}$ let $\mathfrak{z} \mathfrak{a}(\mathfrak{b}):=\{X \in \mathfrak{a}:[X, Y]=0, Y \in \mathfrak{b}\}$ be the centralizer of $\mathfrak{b}$ in $\mathfrak{a}$. We call $(\mathfrak{g}, \tau)$ non-compactly causal, or simply NCC, if $\mathfrak{z} \mathfrak{q} \cap \mathfrak{p}(\mathfrak{h} \cap \mathfrak{k}) \neq\{0\}$. We call $(\mathfrak{g}, \tau)$ non-compactly Riemannian (NCR) if $\tau$ is a Cartan involution. If not otherwise stated from now on $(\mathfrak{g}, \tau)$ denotes a NCC symmetric Lie algebra. Then $\mathfrak{z} \mathfrak{q} \cap \mathfrak{p}(\mathfrak{h} \cap \mathfrak{k})=\mathfrak{z}(\mathfrak{q} \cap \mathfrak{p})=\mathbb{R} X_{0}$ is one dimensional. Let $\mathfrak{a} \subseteq \mathfrak{q} \cap \mathfrak{p}$ be a maximal abelian subspace and note that $\mathbb{R} X_{0} \subseteq \mathfrak{a}$ and that $\mathfrak{a}$ is maximal abelian in $\mathfrak{p}$. We write $\Delta=\Delta(\mathfrak{g}, \mathfrak{a})$ for the root system of $\mathfrak{g}$ with respect to $\mathfrak{a}$ and

$$
\mathfrak{g}=\mathfrak{z} \mathfrak{g}(\mathfrak{a}) \oplus \bigoplus_{\alpha \in \Delta} \mathfrak{g}^{\alpha}
$$

for the corresponding root space decomposition. We write $\mathfrak{g}(0):=\mathfrak{h} \cap \mathfrak{k}+\mathfrak{q} \cap \mathfrak{p}$ and note that $(\mathfrak{g}(0), \tau(0))$, with $\tau(0):=\left.\tau\right|_{\mathfrak{g}(0)}$, is NCR. If $\alpha \in \Delta$ then either $\mathfrak{g}^{\alpha} \subseteq \mathfrak{g}(0)$ or $\mathfrak{g}^{\alpha} \subseteq \mathfrak{q} \cap \mathfrak{k}+\mathfrak{h} \cap \mathfrak{p}$. A root $\alpha \in \Delta$ is called compact if $\mathfrak{g}^{\alpha} \subseteq \mathfrak{g}(0)$ and non-compact if $\mathfrak{g}^{\alpha} \subseteq \mathfrak{q} \cap \mathfrak{k}+\mathfrak{h} \cap \mathfrak{p}$. We write $\Delta_{k}$ and $\Delta_{n}$ for the collection of compact and non-compact roots, respectively. Note that $\Delta=\Delta_{k} \dot{\cup} \Delta_{n}$.

We can and will normalize $X_{0}$ such that $\operatorname{Spec}\left(\operatorname{ad} X_{0}\right)=\{-1,0,1\}$. Then $\Delta_{k}=\{\alpha \in$ $\left.\Delta: \alpha\left(X_{0}\right)=0\right\}$ and we can choose a positive system $\Delta^{+}$of $\Delta$ such that

$$
\Delta_{n}^{+}:=\Delta_{n} \cap \Delta^{+}=\left\{\alpha \in \Delta_{n}: \alpha\left(X_{0}\right)=1\right\}
$$

and such that $\Delta_{k}^{+}:=\Delta_{k} \cap \Delta^{+}$is a positive system in $\Delta_{k}$. Let $\Delta^{-}:=-\Delta^{+}, \Delta_{n}^{-}:=-\Delta_{n}^{+}$and $\Delta_{k}^{-}:=-\Delta_{k}^{+}$.

We recall now few facts about the structure of the root system $\Delta$. Two roots $\alpha, \beta \in \Delta$ are said to be strongly orthogonal if $\alpha \pm \beta$ is not a root. Let $\Gamma:=\left\{\gamma_{1}, \ldots, \gamma_{r}\right\}$ be a system of strongly orthogonal roots in $\Delta_{n}^{+}$of maximal length, i.e., $\Gamma$ consists of pairwise strongly orthogonal roots and has maximal number of elements with respect to this property. We set

$$
\mathcal{W}:=N_{\operatorname{Inn}(\mathfrak{h} \cap \mathfrak{k})}(\mathfrak{a}) / Z_{\operatorname{Inn}(\mathfrak{h} \cap \mathfrak{k})}(\mathfrak{a})
$$

and call $\mathcal{W}$ the small Weyl group of $\Delta$. 
Proposition I.1. For the root system $\Delta=\Delta(\mathfrak{g}, \mathfrak{a})$ of a non-compactly causal symmetric Lie algebra $(\mathfrak{g}, \tau)$ the following assertions hold:

(i) The root system $\Delta$ is reduced, i.e., if $\alpha \in \Delta$ then $2 \alpha \notin \Delta$. In particular, there exists at most two root lengths.

(ii) All long roots in $\Delta_{n}^{+}$are conjugate under the small Weyl group $\mathcal{W}$. Moreover, all roots $\gamma_{i}, 1 \leq i \leq r$, are long.

(iii) Write $\Delta_{n, s}^{+}$for the short roots in $\Delta_{n}^{+}$. Then, if $\Delta_{n, s}^{+} \neq \varnothing$, one has

$$
\Delta_{n, s}^{+}=\left\{\frac{1}{2}\left(\gamma_{i}+\gamma_{j}\right): 1 \leq i<j \leq r\right\}
$$

and all elements of $\Delta_{n, s}^{+}$are conjugate under $\mathcal{W}$.

Proof. (i) [HiÓl96, Th. 3.2.4] or [NÓ99, Lemma 2.12].

(ii) [NÓ99, Lemma 2.26].

(iii) [NÓ99, Lemma 2.22, Lemma 2.24].

For $\alpha \in \Delta$ let $H_{\alpha} \in\left\{[X, \tau(X)]: X \in \mathfrak{g}^{\alpha}\right\} \subseteq \mathfrak{a}$ be such that $\alpha\left(H_{\alpha}\right)=2$. For each $1 \leq i \leq r$ let $H_{i}=H_{\gamma_{i}}$. We set $\mathfrak{c}:=\operatorname{span}_{\mathbb{R}}\left\{H_{1}, \ldots, H_{r}\right\} \subseteq \mathfrak{a}$ and write $\mathfrak{b}$ for the orthogonal complement of $\mathfrak{c}$ in $\mathfrak{a}$ with respect to the Cartan-Killing form; in particular $\mathfrak{a}=\mathfrak{c} \oplus \mathfrak{b}$.

Proposition I.2. The positive system $\Delta_{k}^{+}$can be chsosen such that for the restriction of $\Delta=\Delta(\mathfrak{g}, \mathfrak{a})$ to $\mathfrak{c}$ the following assertions hold:

$$
\begin{gathered}
\left.\Delta_{n}^{+}\right|_{\mathfrak{c}}=\left\{\frac{1}{2}\left(\gamma_{i}+\gamma_{j}\right): 1 \leq i, j \leq r\right\} \cup\left\{\frac{1}{2} \gamma_{i}: 1 \leq i \leq r\right\}, \\
\left.\Delta_{k}^{+}\right|_{\mathfrak{c}} \backslash\{0\}=\left\{\frac{1}{2}\left(\gamma_{i}-\gamma_{j}\right): 1 \leq j<i \leq r\right\} \cup\left\{-\frac{1}{2} \gamma_{i}: 1 \leq i \leq r\right\} .
\end{gathered}
$$

Moreover, the second sets in the two unions from above may or may not occur simultaneously.

Proof. [NÓ99, Th. 2.21] or [Kr99, Th. IV.4].

Since we have free choice for $\Delta_{k}^{+}$we assume in the sequel that $\left.\Delta_{k}^{+}\right|_{\mathfrak{c}} \backslash\{0\} \subseteq\left\{\frac{1}{2}\left(\gamma_{i}-\gamma_{j}\right): 1 \leq\right.$ $j<i \leq r\} \cup\left\{-\frac{1}{2} \gamma_{i}: 1 \leq i \leq r\right\}$.

Lemma I.3. Assume that $\Delta_{n, s} \neq \varnothing$ and let $\Pi_{k}$ be the set of simple roots corresponding to $\Delta_{k}^{+}$. Then there exits $\beta_{1}, \ldots, \beta_{m} \in \mathfrak{b}^{*}, \beta_{j}\left(X_{0}\right)=0$, and $\delta_{1}, \ldots \delta_{l} \in \mathfrak{b}^{*}, \delta_{i}\left(X_{0}\right)=\frac{1}{2}$, such that

$$
\Pi_{k}=\left\{\frac{1}{2}\left(\gamma_{i+1}-\gamma_{i}\right): 1 \leq i \leq r-1\right\} \cup\left\{\beta_{1}, \ldots, \beta_{m}\right\} \cup\left\{-\frac{1}{2} \gamma_{r}+\delta_{i}: 1 \leq i \leq l\right\} .
$$

Here the last set occurs if and only if there exits half roots in $\left.\Delta\right|_{\mathfrak{c}}$.

Proof. For each $\alpha \in \Delta$ let $s_{\alpha}$ denote the corresponding reflection. Then $s_{\gamma_{j}}\left(\frac{1}{2}\left(\gamma_{i}+\gamma_{j}\right)\right)=$ $\frac{1}{2}\left(\gamma_{i}-\gamma_{j}\right), i \neq j$ together with Proposition I.1(iii) shows that $\Delta_{k} \supseteq\left\{\frac{1}{2}\left(\gamma_{i}-\gamma_{j}\right): 1 \leq i \neq j \leq r\right\}$. Thus Proposition I.2 yields that

$$
\begin{aligned}
\Delta_{k}^{+} & \subseteq\left\{\frac{1}{2}\left(\gamma_{i}-\gamma_{j}\right): 1 \leq j<i \leq r\right\}+\left(\mathfrak{b}^{*} \cap X_{0}^{\perp}\right) \\
& \cup\left\{-\frac{1}{2} \gamma_{i}: 1 \leq i \leq r\right\}+\left\{\delta \in \mathfrak{b}^{*}: \delta\left(X_{0}\right)=\frac{1}{2}\right\} \cup \mathfrak{b}^{*} .
\end{aligned}
$$

Now the assertion follows easily from Proposition I.2 and the fact that $\Delta$ is a root system. 
We define the maximal cone in $\mathfrak{a}$ is defined by

$$
C_{\max }:=\left\{X \in \mathfrak{a}:\left(\forall \alpha \in \Delta_{n}^{+}\right) \alpha(X) \geq 0\right\} .
$$

Lemma I.4. Let $X_{0}=X_{0}^{b}+X_{0}^{c}$ with $X_{0}^{b} \in \mathfrak{b}$ and $X_{0}^{c} \in \mathfrak{c}$. Then we have $X_{0}^{b}, X_{0}^{c} \in C_{\max }$.

Proof. First note that $X_{0}^{c}=\frac{1}{2}\left(H_{1}+\ldots+H_{r}\right)$ and so $X_{0}^{c} \in C_{\max }$ by Proposition I.2.

To show $X_{0}^{b} \in C_{\max }$ let $\alpha \in \Delta_{n}^{+}$. Then Proposition I.2 shows that $\alpha=\frac{1}{2}\left(\gamma_{i}+\gamma_{j}\right)+\beta$ with $\beta \in \mathfrak{b}^{*}, \beta\left(X_{0}\right)=\beta\left(X_{0}^{b}\right)=0$, or $\alpha=-\frac{1}{2} \gamma_{i}+\delta$ with $\delta \in \mathfrak{b}^{*}$ and $\delta\left(X_{0}\right)=\delta\left(X_{0}^{b}\right)=\frac{1}{2}$. In any case we have $\alpha\left(X_{0}^{b}\right) \geq 0$ concluding the proof of the lemma.

Finally we define subalgebras of $\mathfrak{g}$ by

$$
\mathfrak{n}:=\bigoplus_{\alpha \in \Delta^{+}} \mathfrak{g}^{\alpha}, \quad \overline{\mathfrak{n}}:=\bigoplus_{\alpha \in \Delta^{-}} \mathfrak{g}^{\alpha}, \quad \mathfrak{n}_{k}^{ \pm}:=\bigoplus_{\alpha \in \Delta_{k}^{ \pm}} \mathfrak{g}^{\alpha}, \quad \mathfrak{n}_{n}^{ \pm}:=\bigoplus_{\alpha \in \Delta_{n}^{ \pm}} \mathfrak{g}^{\alpha}
$$

and note that $\mathfrak{n}=\mathfrak{n}_{n}^{+} \rtimes \mathfrak{n}_{k}^{+}$and $\overline{\mathfrak{n}}=\mathfrak{n}_{n}^{-} \rtimes \mathfrak{n}_{k}^{-}$are semidirect products.

\section{Analytic preliminaries}

Let $G_{\mathbb{C}}$ be a simply connected Lie group with Lie algebra $\mathfrak{g}_{\mathbb{C}}$ and let $G$ be the analytic subgroup of $G_{\mathbb{C}}$ corresponding to $\mathfrak{g}$. Let $H=G^{\tau}=\{X \in G: \tau(g)=g\}$. We write $A, K$, $N, \bar{N}, N_{k}^{ \pm}, N_{n}^{ \pm}$for the analytic subgroups of $G$ which correspond to $\mathfrak{a}, \mathfrak{g}(0), \mathfrak{h}, \mathfrak{k}, \mathfrak{n}, \overline{\mathfrak{n}}$, $\mathfrak{n}_{k}^{ \pm}, \mathfrak{n}_{n}^{ \pm}$. Note that the groups $A, N, \bar{N}, N_{k}^{ \pm}, N_{n}^{ \pm}$are all simply connected and that the corresponding exponential mappings $\exp _{A}: \mathfrak{a} \rightarrow A$, $\exp _{N}: \mathfrak{n} \rightarrow N$ etc. are all diffeomorphisms. Let $G(0)=Z_{G}\left(X_{0}\right)=\left\{g \in G: \operatorname{Ad}(g) \cdot X_{0}=X_{0}\right\}$. Then $H$ and $G(0)$ are $\tau$ and $\theta$ invariant, $H=(H \cap K) \exp (\mathfrak{h} \cap \mathfrak{p})$ and $G(0)=(H \cap K) \exp (\mathfrak{q} \cap \mathfrak{p})$.

The Lie algebra $\mathfrak{g}$ decomposes as $\mathfrak{g}=\mathfrak{h}+\mathfrak{a}+\mathfrak{n}$ and the multiplication mapping

$$
H \times A \times N \rightarrow G, \quad(h, a, n) \mapsto \text { han }
$$

is an analytic diffeomorphism onto its open image $H A N$.

Note that $\bar{N}=N_{n}^{-} \rtimes N_{k}^{-}$. We have

$$
\bar{N} \cap H A N=\exp (\Omega) N_{k}^{-}=N_{k}^{-} \exp (\Omega)
$$

with $\Omega \cong H / H \cap K$ a real bounded symmetric domain in $\mathfrak{n}_{n}^{-}$.

\section{The geometry of the real bounded symmetric domain $\Omega$}

We denote by $\kappa$ the Cartan-Killing form on $\mathfrak{g}$ and define an inner product on $\mathfrak{g}$ by $\langle X, Y\rangle:=-\kappa(X, \theta(Y))$ for $X, Y \in \mathfrak{g}$. Let $X_{i} \in \mathfrak{g}^{\gamma_{i}}$ be such that $H_{i}=\left[X_{i}, X_{-i}\right]$, with $X_{-i}=\tau\left(X_{i}\right)$. By [HiÓl96] and Herman's Convexity Theorem we have

$$
\begin{aligned}
\Omega & =\left\{X \in \mathfrak{n}_{n}^{-}:\|\operatorname{ad}(X+\tau(X))\|<1\right\} \\
& =\operatorname{Ad}(H \cap K) \cdot\left\{\sum_{j=1}^{r} t_{j} X_{-j}:-1<t_{j}<1, j=1, \ldots, r\right\},
\end{aligned}
$$

where $\|\cdot\|$ denotes the operator norm corresponding to the scalar product $\langle\cdot, \cdot\rangle$ on $\mathfrak{g}$. Note that (2.1) implies that $\Omega$ is a convex balanced subset of $\mathfrak{n}_{n}^{-}$. 
Remark II.1. Recall the definition of the maximal cone $C_{r \operatorname{mmax}}$ in $\mathfrak{a}$. Then it is clear from the characterization (2.1) of $\Omega$ that $e^{\operatorname{ad} X} \Omega \subseteq \Omega$ for all $X \in C_{\max }$. We also have a minimal cone in $\mathfrak{a}$ defined by

$$
C_{\min }:=\operatorname{cone}\left(\left\{[X, \tau(X)]: X \in \mathfrak{g}^{\alpha}, \alpha \in \Delta^{+}\right\}\right)=\overline{\sum_{\alpha \in \Delta_{n}^{+}} \mathbb{R}^{+} H_{\alpha}} .
$$

We note that $C_{\min } \subseteq C_{\max }$ and in particular $H_{i} \in C_{\max }$ for each $1 \leq i \leq r$.

The following concept turns out to be very useful for the investigation of the fine convex geomety of $\Omega$.

Definition II.2. (Oshima-Sekiguchi) By a signature of $\Delta$ we understand a map $\varepsilon: \Delta \rightarrow$ $\{-1,1\}$ with the following properties:

(S1) $\varepsilon(\alpha)=\varepsilon(-\alpha)$ for all $\alpha \in \Delta$.

(S2) $\varepsilon(\alpha+\beta)=\varepsilon(\alpha) \varepsilon(\beta)$ for all $\alpha, \beta \in \Delta$ with $\alpha+\beta \in \Delta$.

If $\varepsilon: \Delta \rightarrow\{-1,1\}$ is a signature then $\theta_{\varepsilon}: \mathfrak{g} \rightarrow \mathfrak{g}$ defined by $\theta_{\varepsilon}(X)=\varepsilon(\alpha) \theta(X), X \in \mathfrak{g}^{\alpha}$ and $\left.\theta_{\varepsilon}\right|_{\mathfrak{z}_{\mathfrak{g}}(\mathfrak{a})}=\left.\theta\right|_{\mathfrak{z}_{\mathfrak{g}}(\mathfrak{a})}$ is an involution on $\mathfrak{g}$ that commutes with $\theta$ (see [OS80, Def. 1.2]). As $\left.\tau\right|_{\mathfrak{z}_{\mathfrak{g}}(\mathfrak{a})}=\left.\theta\right|_{\mathfrak{z}_{\mathfrak{g}}(\mathfrak{a})}$ and $\left.\tau\right|_{\mathfrak{g}^{\alpha}}= \pm\left.\theta\right|_{\mathfrak{g}^{\alpha}}$, with + if $\alpha$ is compact and -1 if $\alpha$ non-compact, it follows that $\theta_{\varepsilon}$ also commutes with $\tau$.

Lemma II.3. Keep the notation of Definition II.2.

(i) If $\varepsilon$ is a signature of $\Delta$, then the prescription

$$
\sigma_{\varepsilon}(X):= \begin{cases}X & \text { for } X \in \mathfrak{z}_{\mathfrak{g}}(\mathfrak{a}) \\ \varepsilon(\alpha) X & \text { for } X \in \mathfrak{g}^{\alpha}, \alpha \in \Delta\end{cases}
$$

defines an involutive automorphism of $\mathfrak{g}$. The involution $\sigma_{\varepsilon}$ commutes with both $\tau$ and $\theta$.

(ii) Let $\Pi:=\left\{\alpha_{1}, \ldots, \alpha_{n}\right\}$ be a basis of $\Delta$. Then for any collection $\left(\varepsilon_{1}, \ldots, \varepsilon_{n}\right) \in\{-1,1\}^{n}$ one can define a signature $\varepsilon$ of $\Delta$ by setting

$$
\varepsilon\left( \pm \sum_{i=1}^{n} n_{i} \alpha_{i}\right):=\prod_{i=1}^{n} \varepsilon_{i}^{n_{i}} \quad \text { for } \quad \sum_{i=1} n_{i} \alpha_{i} \in \Delta .
$$

(iii) Let the notation be as in (ii). Then $\varepsilon \mapsto\left(\varepsilon\left(\alpha_{i}\right)\right)_{i=1}^{n}$ defines a bijection between the set of signatures of $\Delta$ and $\{-1,1\}^{n}$.

Proof. (i) This follows by the Oshima-Sekiguchi construction because $\sigma_{\varepsilon}=\tau_{\varepsilon} \theta$. (ii) is clear and (iii) follows from (ii).

In the sequel we identify signatures with elements in $\{-1,1\}^{n}$.

Lemma II.4. Let $\varepsilon$ be a signature of $\Delta$. Then $\sigma_{\varepsilon}(\Omega)=\Omega$.

Proof. Let $X \in \Omega$. By (2.2) there is a $k \in H \cap K$ and $Y=\sum_{j=1}^{r} t_{j} X_{-j},-1<t_{j}<1$ such that $\operatorname{Ad}(k) . Y=X$. As $\sigma_{\varepsilon}$ commutes with $\tau$ and $\theta$ it follows that $\sigma_{\varepsilon}(k) \in K \cap H$. Hence $\sigma_{\varepsilon}(X)=\operatorname{Ad}\left(\sigma_{\varepsilon}(k)\right) \cdot \sum_{j=1}^{r} \varepsilon\left(\gamma_{j}\right) t_{j} X_{-j} \in \Omega$.

Recall that there is basis $\Pi \subseteq \Delta^{+}$having the form

$$
\Pi=\left\{\alpha_{0}, \alpha_{1}, \ldots, \alpha_{n}\right\}
$$

with $\alpha_{0}$ long and non-compact and $\alpha_{i}, 1 \leq i \leq n$ compact. Thus every non-compact negative root $\gamma \in \Delta_{n}^{-}$can be written as $\gamma=-\alpha_{0}-\sum_{i=1}^{n} m_{i} \alpha_{i}, m_{i} \in \mathbb{N}_{0}$. By our choice of $\Delta_{k}^{+}$we have $\alpha_{0}=\gamma_{1}$. 
Theorem II.5. For each $\gamma \in \Delta_{n}^{-}$let $p_{\gamma}: \mathfrak{n}_{n}^{-} \rightarrow \mathfrak{g}^{\gamma}$ be the orthogonal projection. Then

$$
X \in \Omega \Rightarrow p_{\gamma}(X) \in \Omega \text {. }
$$

Proof. Let $X=\sum_{\gamma \in \Delta_{n}^{-}} X_{\gamma} \in \Omega$ with $X_{\gamma} \in \mathfrak{g}^{\gamma}, \gamma \in \Delta_{n}^{-}$. We have to show that $X_{\gamma} \in \Omega$. Recall that there are at most two root length in $\Delta$ (cf. Proposition I.1(i)).

Case 1: $\gamma$ is a long root.

By Proposition I.1(ii) there exists an element $h \in N_{\operatorname{Inn}(\mathfrak{h} \cap \mathfrak{k})}(\mathfrak{a})$ such that $h \cdot \gamma=-\alpha_{0}$. Thus we may assume that $\gamma=-\alpha_{0}=-\gamma_{1}$. Let $H:=\sum_{j=2}^{r} H_{j}$. By Remark II.1 we have

$$
X_{1}:=\lim _{t \rightarrow+\infty} e^{t \text { ad } H} \cdot X \in \Omega .
$$

If we express $X_{1}=\sum_{\beta \in \Delta_{n}^{-}} X_{\beta}$ as a sum of root vectors, then Proposition I.2 implies that $\left.\beta\right|_{\mathfrak{c}}=-\gamma_{1}$ or $\beta=\frac{1}{2} \gamma_{1}-\delta$ with $\delta\left(X_{0}^{b}\right)=\frac{1}{2}$. Since $X_{0}^{b} \in C_{\max }$ (cf. Lemma I.4), we now get

$$
X_{\gamma}=\lim _{t \rightarrow+\infty} e^{t \operatorname{ad} X_{0}^{b}} \cdot X_{1} \in \Omega
$$

Case 2: $\gamma$ is a short root.

By Proposition I.1(iii) we may assume that $\gamma=\frac{1}{2}\left(\gamma_{1}+\gamma_{2}\right)$ and by Lemma I.3 we may suppose $\alpha_{0}=\gamma_{1}, \alpha_{j}=\frac{1}{2}\left(\gamma_{j+1}-\gamma_{j}\right)$ for $1 \leq j \leq r-1$. Write

$$
X=\sum_{m_{i} \geq 0} X_{m_{1}, \ldots, m_{n}},
$$

where $X_{m_{1}, \ldots, m_{n}} \in \mathfrak{g}^{-\left(\alpha_{0}+\sum_{i=1}^{n} m_{i} \alpha_{i}\right)}$. Then we have to show that $X_{1,0, \ldots, 0} \in \Omega$. Set

$$
X_{\mathrm{ev}}:=\sum_{m_{n} \equiv 0(2)} X_{m_{1}, \ldots, m_{n}} \text { and } X_{\mathrm{odd}}:=\sum_{m_{n} \equiv 1(2)} X_{m_{1}, \ldots, m_{n}} .
$$

Then $X=X_{\mathrm{ev}}+X_{\text {odd }}$ and we claim that $X_{\mathrm{ev}}, X_{\mathrm{odd}} \in \Omega$. Let $\varepsilon=(1,1,1, \ldots,-1)$. Then by Lemma II.4 we get:

$$
\sigma_{\varepsilon}(X)=\sigma_{\varepsilon}\left(X_{\mathrm{ev}}+X_{\mathrm{odd}}\right)=X_{\mathrm{ev}}-X_{\mathrm{odd}} \in \Omega .
$$

Since $\Omega$ is balanced and convex we moreover have

$$
X_{\mathrm{ev}}=\frac{1}{2}\left(X+\sigma_{\varepsilon}(X)\right) \in \Omega \quad \text { and } \quad X_{\mathrm{odd}}=\frac{1}{2}\left(X-\sigma_{\varepsilon}(X)\right) \in \Omega .
$$

By repeating this argument we thus my assume that

$$
X=\sum_{\substack{m_{1} \equiv 1(2) \\ m_{j} \equiv 0(2), j>1}} X_{m_{1}, \ldots, m_{n}} .
$$

Now we apply the contraction semigroup generated by $H=\sum_{j=3}^{r} H_{j} \in C_{\max }$ and obtain

$$
X_{1}:=\lim _{t \rightarrow+\infty} e^{t \mathrm{ad} H} \cdot X \in \Omega .
$$

Thus we may assume $X=X_{1}$ and $X=\sum_{\beta \in \Delta_{n}^{-}} X_{\beta}$ with $-\beta=\gamma, \gamma_{1}, \gamma_{2}, \frac{1}{2}\left(\gamma_{1}+\gamma_{2}\right)+$ $\beta,-\frac{1}{2} \gamma_{1}+\sigma_{1},-\frac{1}{2} \gamma_{2}+\sigma_{2}$ and $\beta, \sigma_{1}, \sigma_{2} \in \mathfrak{b}^{*}, \sigma_{1}\left(X_{0}\right)=\sigma_{2}\left(X_{0}\right)=\frac{1}{2}$ (cf. Proposition I.2). Write $\beta=-\gamma_{1}-\sum_{j=1} m_{j} \alpha_{j}$. The cases $\beta=\gamma_{1}$ and $\beta=\gamma_{2}$ are excluded, since we have $m_{1}=0$, resp. $m_{1}=2$, contradicting $m_{1} \equiv 1(2)$. Applying to $X$ the contraction semigroup generated by $X_{0}^{b} \in C_{\max }$ excludes the case $\beta=-\frac{1}{2} \gamma_{1}+\sigma_{1}$ and $\beta=-\frac{1}{2} \gamma_{2}+\sigma_{2}$. Let now $Y \in \mathfrak{b}$ such that $\delta_{j}(Y)>0,1 \leq j \leq l$, and $\beta_{j}(Y)>0,1 \leq j \leq m$ (cf. Lemma I.3). Then $\Delta_{n}^{+} \subseteq \mathbb{N}_{0}[\Pi]$ shows that $Y \in C_{\max }$. But then

$$
X_{\gamma}=\lim _{t \rightarrow+\infty} e^{t \operatorname{ad} Y} \cdot X \in \Omega,
$$

completing the proof Case 2 and hence of the theorem. 


\section{Subdomains of rank one}

For $\alpha \in \Delta^{+}$we set

$$
\mathfrak{g}(\alpha):=\left(\mathfrak{g}^{\alpha}+\mathfrak{g}^{-\alpha}+\left[\mathfrak{g}^{\alpha}, \mathfrak{g}^{-\alpha}\right]\right)^{\prime}
$$

and $\tau(\alpha):=\left.\tau\right|_{\mathfrak{g}(\alpha)}$. Then $(\mathfrak{g}(\alpha), \tau(\alpha))$ is a symmetric subalgebra of $(\mathfrak{g}, \tau)$ of real rank one, that is $\mathfrak{a}(\alpha):=\mathfrak{a} \cap \mathfrak{g}(\alpha)$ is one dimensional. Further we set $\mathfrak{h}(\alpha):=\mathfrak{h} \cap \mathfrak{g}(\alpha)$ etc. We denote by $G(\alpha), A(\alpha)$ etc. the analytic subgroups of $G$ corresponding to $\mathfrak{g}(\alpha), \mathfrak{a}(\alpha)$ etc. Let $H(\alpha)=G(\tau)^{\tau(\alpha)}=G(\alpha) \cap H$.

Assume that $\alpha \in \Delta_{n}^{+}$. Then $(\mathfrak{g}(\alpha), \tau(\alpha))$ is NCC and $\mathfrak{n}(\alpha)=\mathfrak{n}_{n}^{+}(\alpha)=\mathfrak{g}^{\alpha}$. Let $\Omega(\alpha) \cong H(\alpha) /(K(\alpha) \cap H(\alpha))$ be the real bounded symmetric domain in $\overline{\mathfrak{n}}(\alpha)=\mathfrak{n}_{n}^{-}(\alpha)$.

Lemma II.6. Let $\alpha \in \Delta_{n}^{+}$and $s_{\alpha} \in G(\alpha)$ be a representaive of the one element big Weyl group $N_{G(\alpha)}(\mathfrak{a}(\alpha)) / Z_{G(\alpha)}(\mathfrak{a}(\alpha))$ of $\mathfrak{g}(\alpha)$. Then

$$
(\bar{N}(\alpha) \cap H(\alpha) A(\alpha) N(\alpha)) \dot{\cup}\left(\bar{N}(\alpha) \cap H(\alpha) s_{\alpha} A(\alpha) N(\alpha)\right)
$$

is open and dense in $\bar{N}(\alpha)$.

Proof. This follows by Matsukis Theorem (cf. [Ma79, Theorem 3]), if we can show that $M(\alpha):=Z_{K(\alpha)}(\mathfrak{a}(\alpha)) \subseteq H(\alpha)$ because $s_{\alpha} M(\alpha)=M(\alpha) s_{\alpha}$. Let $F=\exp (i \mathfrak{a}(\alpha)) \cap G(\alpha)$. Then one has $M(\alpha)=F Z_{H(\alpha)_{o}}(\mathfrak{a}(\alpha))$ by [NÓ99, Lemma 5.7]. But if $f \in F$ then $\tau(\alpha)(f)=f^{-1}=f$, by the same lemma. Hence $F \subseteq H(\alpha)$, which implies that $M(\alpha) \subseteq H(\alpha)$.

Theorem II.7. Let $\alpha \in \Delta_{n}^{+}$. Then $\Omega \cap \overline{\mathfrak{n}}(\alpha)=\Omega(\alpha)$.

Proof. "卫": This is clear.

" $\subseteq$ : Note that $\Omega \cap \overline{\mathfrak{n}}(\alpha)$ is open and convex in $\overline{\mathfrak{n}}(\alpha)$. We have

$$
\exp (\Omega) \cap\left(H(\alpha) s_{\alpha} A(\alpha) N(\alpha)\right)=\varnothing,
$$

since $\exp (\Omega) \subseteq H A N$ and $H A N \cap H s_{\alpha} A N=\varnothing$ by Matsukis Theorem. In view of (2.3), Lemma II.7 implies that there exists an open dense subset $\Omega_{\alpha}$ of $\Omega \cap \overline{\mathfrak{n}}(\alpha)$ such that $\Omega_{\alpha} \subseteq \Omega(\alpha)$. Now the assertion follows from the fact that both $\Omega(\alpha)$ and $\Omega \cap \overline{\mathfrak{n}}(\alpha)$ are open and convex.

\section{The product formula for the $c$-function}

Recall the $H A N$-decomposition in $G$ from Section I. For each $\lambda \in \mathfrak{a}_{\mathbb{C}}^{*}$ and $g$ in $G$ we set

$$
a_{H}(g)^{\lambda}:=\left\{\begin{array}{ll}
0 & \text { if } g \notin H A N \\
e^{\lambda(\log a)} & \text { if } g=\text { han } \in H A N
\end{array} .\right.
$$

For a locally compact group $G$ we write $\mu_{G}$ for a left Haar measurwe on $G$.

Definition III.1. (The $c$-functions) For each $\alpha \in \mathfrak{a}^{*}$ let $m_{\alpha}:=\operatorname{dim} \mathfrak{g}^{\alpha}$ and put $\rho:=$ $\frac{1}{2} \sum_{\alpha \in \Delta^{+}} m_{\alpha} \alpha$. For $\lambda \in \mathfrak{a}_{\mathbb{C}}^{*}$ we now set

$$
c(\lambda):=\int_{\bar{N}} a_{H}(\bar{n})^{-(\lambda+\rho)} d \mu_{\bar{N}}(\bar{n})=\int_{\bar{N} \cap H A N} a_{H}(\bar{n})^{-(\lambda+\rho)} d \mu_{\bar{N}}(\bar{n}),
$$




$$
c_{\Omega}(\lambda):=\int_{N_{n}^{-}} a_{H}(\bar{n})^{-(\lambda+\rho)} d \mu_{N_{n}^{-}}(\bar{n})=\int_{\Omega} a_{H}(\bar{n})^{-(\lambda+\rho)} d \mu_{N_{n}^{-}}(\bar{n}),
$$

and

$$
c_{0}(\lambda):=\int_{N_{k}^{-}} a_{H}(\bar{n})^{-(\lambda+\rho)} d \mu_{N_{k}^{-}}(\bar{n})
$$

whenever the defining integral exist. We write $\mathcal{E}, \mathcal{E}_{\Omega}$ and $\mathcal{E}_{0}$ for the domain of definition of $c$, $c_{\Omega}$ and $c_{0}$, respectively. We call $c$ the $c$-function of the non-compactly causal symmetric space $G / H$ and $c_{\Omega}$ the $c$-function of the real bounded symmetric domain $\Omega$, while $c_{0}$ is the usual c-function of the non-compact Riemannian symmetric space $G(0) / K(0)$.

Remark III.2. (a) The choice of the particular analytic realization $G / H$ of $(\mathfrak{g}, \tau)$ as a symmetric space is immaterial for the definition of the $c$-function.

(b) We have $\mathcal{E}=\mathcal{E}_{0} \cap \mathcal{E}_{\Omega}$ and for all $\lambda \in \mathcal{E}$ one has the splitting

$$
c(\lambda)=c_{0}(\lambda) c_{\Omega}(\lambda)
$$

(cf. [FHÓ94, Lemma 9.2]).

(c) The $c$-functions can be written as Laplace transforms (cf. [KNÓ98]). Let us explain this for the $c$-function $c$. For $c_{0}$ and $c_{\Omega}$ one has analogous statements.

There exists a positive Radon measure $\mu$ on $\mathfrak{a}$ such that

$$
(\forall \lambda \in \mathcal{E}) \quad c(\lambda)=\mathcal{L}_{\mu}(\lambda):=\int_{\mathfrak{a}} e^{\lambda(X)} d \mu(X),
$$

i.e., $c$ is the Laplace transform of $\mu$. In particular we see that the domain of definition $\mathcal{E}$ is a tube domain over a convex set, i.e., one has

$$
\mathcal{E}=i \mathfrak{a}^{*}+\mathcal{E}_{\mathbb{R}}
$$

with $\mathcal{E}_{\mathbb{R}} \subseteq \mathfrak{a}^{*}$ a convex subset of $\mathfrak{a}^{*}$. One knows that int $\mathcal{E}$ is non-empty. Moreover, the fact that $c$ is a Laplace transform implies that $c$ is holomorphic on $\operatorname{int} \mathcal{E}$ and that $c$ has no holomorphic extension to a connected open tube domain strictly larger than int $\mathcal{E}$.

Now we are going to prove the product formula for the $c$-function $c_{\Omega}$. Our srategy is a modified Gindikin-Karpelevic approach as presented in [GaVa88, p. 175-177] or [Hel84, Ch. IV].

For a positive system $R \subseteq \Delta$ we set $\overline{\mathfrak{n}}_{R}:=\bigoplus_{\alpha \in-\left(\Delta^{+} \cap R\right)} \mathfrak{g}^{\alpha}$ and write $\bar{N}_{R}$ for the corresponding analytic subgroup of $G$. We define an auxiliary $c$-function by

$$
c_{R}(\lambda):=\int_{\bar{N}_{R}} a_{H}(\bar{n})^{-(\lambda+\rho)} d \mu_{\bar{N}_{R}}(\bar{n})
$$

whenever the integral exists.

For a single root $\alpha \in \Delta^{+}$we set $\rho_{\alpha}:=\frac{1}{2} m_{\alpha} \alpha$ and write

$$
c_{\alpha}(\lambda):=\int_{\bar{N}(\alpha)} a_{H(\alpha)}(\bar{n})^{-\left(\lambda+\rho_{\alpha}\right)} d \mu_{\bar{N}(\alpha)}(\bar{n}) .
$$

We denote by $\mathcal{E}_{\alpha} \subseteq \mathfrak{a}_{\mathbb{C}}^{*}$ the domain of definition of $c_{\alpha}$. 
Proposition III.3. For any positive system $R \subseteq \Delta$ we have that

$$
c_{R}(\lambda)=\prod_{\alpha \in\left(R \cap \Delta^{+}\right)} c_{\alpha}(\lambda)
$$

and $c_{R}(\lambda)$ is defined if and only if $\lambda \in \bigcap_{\alpha \in\left(R \cap \Delta^{+}\right)} \mathcal{E}_{\alpha}$.

Proof. We proceed by induction on $\left|R \cap \Delta^{+}\right|$. If $R \cap \Delta^{+}=\varnothing$, then the assertion is clear.

Assume that $R \cap \Delta^{+} \neq \varnothing$. Then we find an element $\beta \in R \cap \Delta^{+}$which is simple in $R$. Set $Q:=s_{\beta} . R$. Then $Q=R \backslash\{\beta\} \cup\{-\beta\}$ since $\Delta$ is reduced (cf. Proposition I.1(i)). Thus we have $\left(Q \cap \Delta^{+}\right) \dot{\cup}\{\beta\}=R \cap \Delta^{+}$. We now have to distinguish to cases.

Case 1: $\beta$ is compact.

In this case, the $H A N$-decomposition of $G(\beta)$ coincides with the Iwasasa decomposition, i.e. $G(\beta)=K(\beta) A(\beta) N(\beta)$. Thus $c_{R}(\lambda)=c_{\beta}(\lambda) c_{Q}(\lambda)$ follow as in [GaVa88, Prop. 4.7.6].

Case 2: $\beta$ is non-compact.

Set $\bar{N}_{Q}^{k}:=\bar{N}_{Q} \cap N_{k}^{-}, \bar{N}_{Q}^{n}:=\bar{N}_{Q} \cap N_{n}^{-}$and note that $\bar{N}_{Q}=\bar{N}_{Q}^{n} \rtimes \bar{N}_{Q}^{k}$. Since $\bar{N}_{R}=\bar{N}(\beta) \bar{N}_{Q}$ we thus get

$$
c_{R}(\lambda)=\int_{\bar{N}_{(\beta)}} \int_{\bar{N}_{Q}^{n}} \int_{\bar{N}_{Q}^{k}} a_{H}\left(\bar{n}_{\beta} \bar{n}_{n} \bar{n}_{k}\right)^{-(\lambda+\rho)} d \mu_{\bar{N}(\beta)}\left(\bar{n}_{\beta}\right) d \mu_{\bar{N}_{Q}^{n}}\left(\bar{n}_{n}\right) d \mu_{\bar{N}_{Q}^{k}}\left(\bar{n}_{k}\right) .
$$

If $\bar{n}_{\beta} \bar{n}_{n} \bar{n}_{k} \in \bar{N} \cap H A N$, then (1.1) implies that $\bar{n}_{\beta} \bar{n}_{n} \in \exp (\Omega)$. Since $\mathfrak{n}_{n}^{-}$is abelian, Theorem II.5 therefore implies that $\bar{n}_{\beta} \in \exp (\Omega)$ and so $\bar{n}_{\beta} \in \exp (\Omega(\beta))$ by Theorem II.7. Therefore we can write $\bar{n}_{\beta}=h_{\beta} a_{\beta} n_{\beta}$ with $h_{\beta} \in H(\beta), a_{\beta} \in A(\beta)$ and $n_{\beta} \in N(\beta)$. Now one can proceed as in [GaVa88, p. 175-177] and one gets $c_{R}(\lambda)=c_{\beta}(\lambda) c_{Q}(\lambda)$.

Remark III.4. If we choose $R=-\Delta_{n}^{+} \cup \Delta_{k}^{+}$(this is a positive system since $\Delta_{n}^{+}$is $\mathcal{W}$ invariant), then we have $c_{0}=c_{R}$ and Proposition III.3 results in the Gindikin-Karpelevic product formula

$$
c_{0}(\lambda)=\prod_{\alpha \in \Delta_{k}^{+}} c_{\alpha}(\lambda)
$$

of the $c$-function $c_{0}$ on $G(0) / K(0)$ (cf. [GaVa88, Th. 4.7.5] or [Hel84, Ch. IV, Th. 6.13, 6.14])

Theorem III.5. (The product formula for $c_{\Omega}$ ) For the c-function $c_{\Omega}$ of the real bounded symmetric domain $\Omega$ one has

$$
\mathcal{E}_{\Omega}=\left\{\lambda \in \mathfrak{a}_{\mathbb{C}}^{*}:\left(\forall \alpha \in \Delta_{n}^{+}\right) \operatorname{Re} \lambda\left(H_{\alpha}\right)<2-m_{\alpha}\right\}
$$

and

$$
c_{\Omega}(\lambda)=\kappa \prod_{\alpha \in \Delta_{n}^{+}} B\left(\frac{m_{\alpha}}{2},-\frac{\lambda\left(H_{\alpha}\right)}{2}-\frac{m_{\alpha}}{2}+1\right)
$$

where $B$ denotes the Beta function and $\kappa$ is a positive constant only depending on $(\mathfrak{g}, \tau)$.

Proof. Set $\mathcal{E}_{\Omega}^{\prime}:=\bigcap_{\alpha \in \Delta_{n}^{+}} \mathcal{E}_{\alpha}$. We want to apply Proposition III.3 to $R=\Delta^{+}$. In view of Remark III.2(b) and Remark III.4, we thus get

$$
\left(\forall \lambda \in \mathcal{E} \cap \mathcal{E}_{\Omega}^{\prime}\right) \quad c_{\Omega}(\lambda)=\prod_{\alpha \in \Delta_{n}^{+}} c_{\alpha}(\lambda)=\prod_{\alpha \in \Delta_{n}^{+}} c_{\Omega(\alpha)}(\lambda) .
$$


By [FHÓ94, (10.3)] one has

$$
c_{\Omega(\alpha)}(\lambda)=2^{m_{\alpha}-1} B\left(\frac{m_{\alpha}}{2},-\frac{\lambda\left(H_{\alpha}\right)}{2}-\frac{m_{\alpha}}{2}+1\right)
$$

and

$$
\mathcal{E}_{\Omega(\alpha)}=\left\{\lambda \in \mathfrak{a}_{\mathbb{C}}^{*}: \operatorname{Re} \lambda\left(H_{\alpha}\right)<2-m_{\alpha}\right\}
$$

It follows from (3.3) that

$$
\mathcal{E}_{\Omega}^{\prime}=\left\{\lambda \in \mathfrak{a}_{\mathbb{C}}^{*}:\left(\forall \alpha \in \Delta_{n}^{+}\right) \operatorname{Re} \lambda\left(H_{\alpha}\right)<2-m_{\alpha}\right\} .
$$

Besides $\mathcal{E}_{\Omega}=\mathcal{E}_{\Omega}^{\prime}$ all assertions of the theorem now follow from (3.1)-(3.4). Finally, $\mathcal{E}_{\Omega}=\mathcal{E}_{\Omega}^{\prime}$ follows from the fact that all $c$-functions involved are Laplace transforms (cf. Remark III.2(c)).

The following simple fact that shows that we can split off all the non-compact roots to get the $c_{\Omega}$-function before we come to the compact roots.

Lemma III.6. Let $R$ be a any positive system of roots in $\Delta$. If $R \cap \Delta_{n}^{+} \neq \varnothing$, then $R \cap \Delta_{n}^{+}$ contains a a root that is simple in $R$.

Proof. Let $\left\{\beta_{0}, \ldots, \beta_{n}\right\}$ be the set of simple roots in $R$. Let $\gamma \in R \cap \Delta_{n}^{+}$. Then $\gamma=\sum_{i=0}^{n} n_{i} \beta_{i}$ with $n_{i} \in \mathbb{N}_{0}$. Thus $1=\gamma\left(X_{0}\right)=\sum_{i=0}^{n} n_{i} \beta_{i}\left(X_{0}\right)$ which implies that $\beta_{i}\left(X_{0}\right)>0$ for at least one $\beta_{i}$. But then $\beta_{i} \in \Delta_{n}^{+}$.

\section{References}

[Fa95] Faraut, J., Fonctions Sphériques sur un Espace Symétrique Ordonné de Type Cayley, Contemp. Math. 191 (1995), 41-55.

[FHÓ94] Faraut, J., J. Hilgert, and G. Ólafsson, Spherical functions on ordered symmetric spaces, Ann. Inst. Fourier 44 (1994), 927-966.

[GaVa88] Gangolli, R., and V.S. Varadarajan, "Harmonic Analysis of Spherical Functions on Real Reductive Groups," Ergebniss der Mathematik 101, Springer, 1988.

[Gr97] Graczyk, P., Function c on an ordered symmetric space, Bull. Sci. math. 121 (1997), 561-572.

[Hel84] Helgason, S., "Groups and Geometric Analysis", Acad. Press, London, 1984.

[HiÓl96] Hilgert, J. and G. Ólafsson, "Causal Symmetric Spaces, Geometry and Harmonic Analysis," Acad. Press, 1996.

[Kr99] Krötz, B., Formal dimension of semisimple symmetric spaces, Compositio math., to appear.

[KNÓ98] Krötz, B., K.-H. Neeb, and G. Ólafsson, Spherical Functions on Mixed Symmetric Spaces, submitted.

[Ma79] Matsuki, T., The orbits of affine symmetric spaces under the action of minimal parabolic subgroups, J. Math. Soc. Jpn. 31, 331-357 (1979).

[NÓ99] Neumann, A., and G. Ólafsson, Minimal and Maximal Semigroups Related to Causal Symmetric Spaces, Semigroup Forum, to appear.

[Ól97] Ólafsson, G., Spherical Functions and Spherical Laplace Transform on Ordered Symmetric Spaces, submitted. 
[OS80] Oshima, S., Sekiguchi, J, Eigenspaces of Invariant Differential Operators on an Affine Symmetric Spaces, Invent. math. 57 (1980), 1-81.

Bernhard Krötz

Mathematical Institute

TU Clausthal

Erzstraße 1

D-38678 Clausthal-Zellerfeld

Germany

e-mail: mabk@math.tu-clausthal.de
Gestur Ólafsson

Department of Mathematics

Louisiana State University

Baton Rouge

LA 70803

e-mail: olafsson@math.lsu.edu 